LIVER

\title{
Evaluation of a prison outreach clinic for the diagnosis and prevention of hepatitis C: implications for the national strategy
}

\author{
C Skipper, J M Guy, J Parkes, P Roderick, W M Rosenberg
}

Gut 2003;52:1500-1504

See end of article for authors' affiliations

.....................

Correspondence to: Dr W M C Rosenberg, Liver Group, Division of Infection, Inflammation, and Repair, Mailpoint 811, Level D South Block Southampton General Hospital, Tremona Rd, Southampton, Hampshire SO166YD, UK; wmr@ soton.ac.uk

Accepted for publication 9 June 2003
Background: Hepatitis $\mathrm{C}$ virus (HCV) infection is a major public health problem recognised by the UK National Strategy that proposes that a care pathway for assessment, diagnosis, and treatment be established in all prisons, integrated within managed clinical networks. A prison sentence provides the opportunity to focus on traditionally hard to reach patients.

Aims: To evaluate the prevalence of HCV infection in a UK prison cluster and to assess the effectiveness of a prison outreach service for hepatitis $C$.

Subjects: Male prisoners.

Methods: A nurse specialist led clinic within a cluster of adult prisons was established, offering health education on hepatitis C, advice on harm minimisation, and HCV testing. Infected prisoners were offered access to a care pathway leading to treatment. Outcome measures were uptake of the service, and diagnosis and treatment of hepatitis $C$.

Results: A total of $8.5 \%$ of 1618 prisoners accepted testing: $30 \%$ had active infection with HCV. Most were ineligible for treatment due to psychiatric illness or did not receive treatment for logistic reasons. Injecting drug use was the major risk factor in all cases. Only $7 \%$ of HCV polymerase chain amplification positive inmates received treatment in prison.

Conclusion: There is a large pool of HCV infected prisoners at risk of complications, constituting a source of infection during their sentence and after discharge. A prison outreach clinic and care pathway was perceived as effective in delivering health education, reducing the burden on prison and hospital services. It provided an opportunity for intervention but had a limited effect in eradicating HCV in prisoners and it remains unclear how this might be achieved.
$\mathrm{H}$ epatitis $\mathrm{C}$ virus (HCV) infection is increasingly recognised as a major health care problem throughout the world. In the UK, HCV is thought to infect at least 200000 people, although the majority are unrecognised. ${ }^{1}$ Estimates of prevalence range from $0.02 \%{ }^{2}$ in blood donors to $0.8 \%^{3}$ among inner city antenatal clinic attendees. The variation in estimates of prevalence may be attributable largely to the variable inclusion of high risk groups, as well as biases in subject selection and differences in the methods of testing for HCV. Transmission is through parenteral exposure to infected blood and infection is known to be clustered at high levels in injecting drug users (IDU). Surveys of HCV infection among IDU have reported prevalence rates as high as $70-90 \%{ }^{45}$ among habitual injectors.

Due to the illegality of injecting drug use and the close association between crime and drug manufacture, trafficking, supply, and use, those involved with IDU frequently pass through the penal system and spend time in prisons. Transmission of HCV within prisons is known to occur through behaviours such as illicit drug use and possibly through body adornment. However, the frequency with which transmission occurs is not known. The prison population is both at high risk for HCV infection and forms a discrete population within which prevalence can be estimated. It is also an opportunity to focus health care on a traditionally hard to reach group.

In 2000 there were 137 prisons in England and Wales, with an average population of 64600 inmates of which 49636 (77\%) were men: $15 \%$ of men and $37 \%$ of women had been sentenced for drug offences but drugs may have contributed to the offences committed by many others. ${ }^{6}$ The prison population is by no means stable as there is an annual throughput of 200000 persons per year, reflecting a very high turnover rate, particularly among those on remand. The average length of sentence in 2000 was 24 months but the average length of stay for a prisoner in any one prison was only six months.

Anonymous testing of prisoners for hepatitis $\mathrm{C}$ has been regarded as unethical in the UK. Studies of prisoners volunteering for testing have reported rates of infection as low as $7 \%$, even in IDU. ${ }^{7}$ In contrast, a survey employing anonymous unlinked testing reported a prevalence of $37 \%$ in Irish prisoners. ${ }^{8}$ Increasing emphasis on the civil rights of prison inmates and initiatives that seek equity of access to healthcare for prisoners have highlighted the need for accurate estimates of the prevalence of HCV in prisons.

The consultation document "Patient or prisoner", published in 1996, emphasised the right of prisoners to the same level of healthcare as that provided to society at large and led to reforms that culminated in the formation of the Prison Directorate of Health Care to oversee this process. The recent National Strategy for Hepatitis C has identified the problem of IDUs passing through prisons and proposed that information about hepatitis $\mathrm{C}$ should be provided to all new admissions to prisons and harm minimisation policies introduced, especially in young offenders institutions.

\footnotetext{
Abbreviations: $\mathrm{HCV}$, hepatitis $\mathrm{C}$ virus; IDU, injecting drug users; $\mathrm{HWC}$, healthwatch clinic; POC, prison outreach clinic; HIV, human immunodeficiency virus; PCR, polymerase chain reaction amplification
} 
Moreover, prisoners should have access to appropriate clinical investigation and NHS treatment for hepatitis C. It is proposed that within managed clinical networks that care pathways are established for prisoners, and that primary care trusts would be expected to have such plans where appropriate.

This paper presents an evaluation of an innovative prison outreach service linked to a specialist hepatology unit for the Isle of Wight cluster of prisons. It describes the prevalence of hepatitis $C$ and the likely impact of a care pathway to assess and treat such patients on the burden of infection.

\section{METHODS}

\section{Setting}

The Isle of Wight prison cluster comprises HMP Parkhurst, HMP Albany, and HMP Camp Hill. All three are category B prisons. Parkhurst detains long and medium stay prisoners as well as some on remand. Albany has a high proportion of prisoners sentenced for sex offences. Demographic data and the percentage of prisoners sentenced for drugs offences are presented in table 1. Camp Hill has a large number of prisoners on remand and a relatively high proportion of prisoners sentenced for drugs offences. Characteristics of the three prison populations are shown in table 1 .

\section{Intervention}

In 1997, the healthwatch clinic (HWC) was established by one of the authors (CS) to provide counselling on blood borne viruses for all new receptions to the Isle of Wight prisons. The prison outreach clinic (POC) of Southampton University Hospitals Trust Liver Group was established by two of the authors (WR and JG) in 1999 in an attempt to improve the quality of care offered to the increasing number of prisoners referred from the Isle of Wight prison cluster. Protocols for the diagnosis and management of inmates with antibodies to HCV were established. An overview of the care pathway is presented in figure 1.

\section{The healthwatch clinic}

All prisoners new to the Isle of Wight prison cluster attend a one hour health awareness lecture during which they are alerted to the risk factors for blood borne viruses. Prisoners are invited to attend the HWC. This clinic provides detailed counselling on blood borne viruses for all new receptions to the Isle of Wight prisons and offers confidential testing for antibodies to hepatitis viruses and human immunodeficiency virus (HIV). Pretest counselling is provided by a nurse specialist and the results of the tests are fed back to prisoners by this nurse or the prison doctor in the context of post-test counselling. Patients identified as having a positive antibody

Table 1 Demographic and sentencing characteristics of the inmates in the three Isle of Wight prisons during the study period. Characteristics are give as a percentage of the prison population mean for 2000-2001

\begin{tabular}{lllll}
\hline & Overall \% & Parkhurst & Camp Hill & Albany \\
\hline Age $<30$ years & 37.2 & 51.5 & 45.2 & 11 \\
Drugs offences & 19.6 & 30.7 & 24.6 & 0.5 \\
White & 69.7 & - & - & - \\
Black & 24.1 & - & - & - \\
Asian & 2.9 & - & - & - \\
Other & 3.3 & - & - & - \\
\hline
\end{tabular}

The data presented are means for the year and refer to current established inmates as well as new receptions. Although the prison populations change, the prisoners captured in these data are representative of those prisoners invited to attend the healthwatch clinic. A breakdown of the ethnic groupings represented in all three prisons is presented.

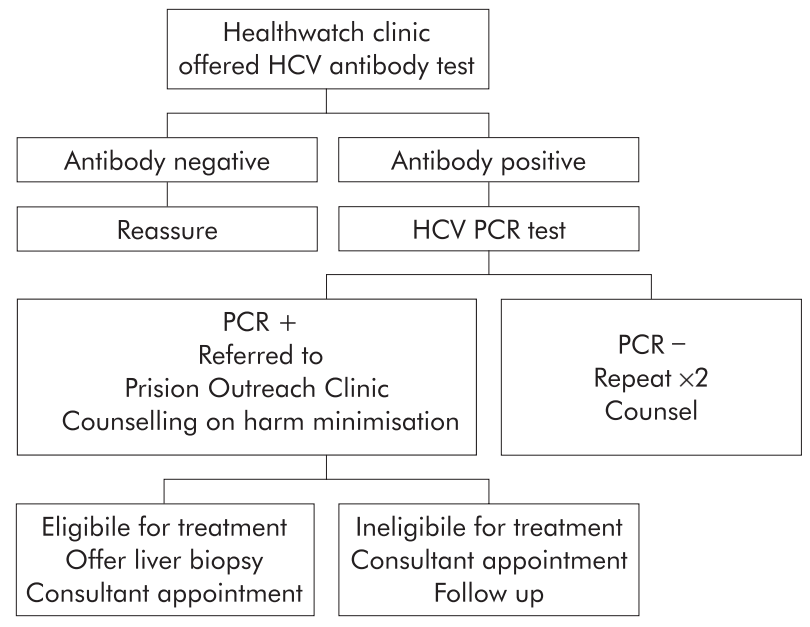

Figure 1 Flow chart depicting the management algorithm for hepatitis $\mathrm{C}$ virus (HCV) testing in the Isle of Wight Prisons. Prisoners were offered the opportunity to make appointments for confidential testing for antibodies to hepatitis viruses and human immunodeficiency virus. Pretest counselling was provided by the prison nurse, with the results fed back to prisoners by the prison nurse or a health advisor with post test counselling. Patients found to have a positive antibody test for HCV were informed that they had evidence of contact with HCV. A polymerase chain amplification (PCR) test for the presence of HCV viral RNA as a marker of ongoing infection was offered and a blood sample was obtained to test liver function. Prisoners positive for HCV by antibody testing but negative on a single PCR test were offered two further PCR tests. If repeated PCR tests were negative, the prisoner was informed that they were not currently infected with $\mathrm{HCV}$ and they were offered the opportunity of a consultation in the prison outreach clinic (POC).

Prisoners who tested positive for HCV RNA were informed that they had ongoing $\mathrm{HCV}$ infection. They were counselled on harm minimisation with respect to alcohol consumption and continued injecting drug use, sharing razors and tooth brushes, personal grooming equipment, and sex. All patients testing positive for HCV by PCR were offered an appointment in the POC. New inmates were seen by a hepatology clinical nurse specialist, a clinical history obtained, and the results of the tests performed explained. The consultations follow a prescribed protocol and cover HCV transmission; the natural history of hepatitis $\mathrm{C}$, including modifying factors and harm minimisation; indications for treatment; and the outcome of treatment, including beneficial and harmful effects of therapy. All patients with a positive HCV PCR test were considered eligible for a liver biopsy in order to determine prognosis and indication for therapy on the basis of the histological severity of liver disease. Patients were counselled about the indications for liver biopsy and the relevance of the histological findings in determining prognosis and taking decisions about treatment. Investigations were described and patients were informed of the adverse effects and complications of liver biopsy. Patients were considered to be ineligible for liver biopsy if they declined the offer of biopsy, if they had a history of significant psychiatric morbidity, or if they expressed the intention to return to chaotic drug or alcohol use or admitted to continuing drug use. Prior to liver biopsy, patients were seen in prison by the consultant at the POC and indications for biopsy were reviewed. Patients were subsequently transferred to Southampton University Hospital Trust for ultrasound guided liver biopsies which were performed during an overnight stay. The results of the biopsies were fed back to the patients by the consultant at the POC. The implications of the biopsy findings were discussed and indications for treatment were reviewed. Patients eligible for therapy were then offered treatment in prison where appropriate. For prisoners about to be released or seeking transfer, referral was made to an appropriate local specialist. Patients who were not eligible for a biopsy or therapy were offered annual follow up appointments in the POC. On release, all patients who had attended the POC were offered referral to a local specialist.

test for HCV are informed that they have evidence of contact with HCV and they are offered a polymerase chain amplification (PCR) test for the presence of HCV viral RNA as a marker of ongoing infection. At the same time, a blood sample is obtained to test serum biochemical markers of liver function. Prisoners who are positive for HCV by antibody 
testing but negative on a single PCR test are offered two further PCR tests. If repeated PCR tests are negative, the prisoner is informed that they are not currently infected with HCV and they are offered the opportunity of a consultation in the POC

Prisoners who test positive for HCV RNA are informed that they have ongoing HCV infection. They are counselled on harm reduction and harm minimisation with respect to alcohol consumption and continued injecting drug use, sharing razors and toothbrushes, personal grooming equipment, and sex. All patients testing positive for HCV by PCR are offered an appointment in the POC.

\section{The POC}

In the POC, prisoners are seen by a hepatology clinical nurse specialist. A clinical history is obtained and the results and interpretation of the tests are reviewed and explained. The consultations follow a prescribed protocol and cover $\mathrm{HCV}$ transmission; the natural history of hepatitis $\mathrm{C}$, including modifying factors and harm minimisation; indications for treatment; and the outcome of treatment, including the beneficial and harmful effects of therapy. All patients with a positive HCV PCR test are considered eligible for a liver biopsy in order to determine prognosis and indications for therapy on the basis of the histological severity of liver disease. Patients are counselled about the indications for liver biopsy and the relevance of the histological findings in determining prognosis and taking decisions about treatment. The investigations are described and patients are informed of the adverse effects and complications of liver biopsy. Patients are considered to be ineligible for liver biopsy if they decline the offer of biopsy, if they have a history of significant psychiatric morbidity, including suicidal ideation, recent deliberate self harm, and psychotic behaviour, or if they express the intention to return to chaotic drug use or admit to continuing drug use in prison.

Prior to liver biopsy, patients are seen in prison by the consultant in the POC and the indications for biopsy are reviewed. Patients are subsequently transferred to Southampton General Hospital for ultrasound guided liver biopsies which are performed during an overnight stay. The results of the biopsies are fed back to the patients by the consultant in the POC. The implications of the biopsy findings are discussed and indications for treatment are reviewed.

Patients eligible for therapy are then offered further screening and treatment is delivered in prison where appropriate. For prisoners about to be released or seeking transfer, referral is made to an appropriate local specialist. Patients who are not eligible for a biopsy or therapy are offered annual follow up appointments in the POC. On release, all patients who have attended the POC are offered referral to a local specialist.

We report on the experience of one year of new inmates entering the Isle of Wight prisons in 2000-2001.

\section{RESULTS}

A total of 1618 prisoners entered the three prisons in the one year period, all of whom received an initial general health lecture: 137 (8.5\%) prisoners requested testing for HCV after initial counselling. The highest rate of requests for testing was recorded in Parkhurst, which has the highest percentage of prisoners sentenced for drugs offences. The fewest requests were made in Albany that holds a large proportion of inmates sentenced for sex offences (tables 1,2 ).

Fifty eight of $137(42 \%)$ inmates tested were found to have a positive HCV antibody test and 41 (30\%) had detectable HCV by PCR, denoting ongoing infection, representing $71 \%$ of antibody positive inmates.

\section{Risk factors}

All antibody positive inmates $(n=58)$ reported at least one potential exposure. Fifty six (97\%) admitted to having injected drugs; $19(33 \%)$ while in prison. Forty one $(71 \%)$ were tattooed. Five $(9 \%)$ reported having received a blood transfusion prior to 1991. In one subject intranasal drug use was the only identifiable potential exposure to HCV and none of the inmates identified sex with a HCV positive partner as their only exposure to the virus.

Twenty four of 58 seropositive inmates reported that they had been tested prior to the present study. However, seven could not recall the setting in which the test had been undertaken. Five had been tested in other prisons, four in hospital, four in drug rehabilitation centres, three in genitourinary medicine clinics, and one by his general practitioner. Few were certain of their previous test results or their significance, findings similar to those of a previous study. ${ }^{9}$ All but one were PCR positive. It is not known how many inmates believed themselves to be $\mathrm{HCV}$ positive erroneously but at least one volunteer for testing was adamant that he had been told that he had active infection but he proved to carry neither HCV nor antibodies to the virus.

\section{Treatment care pathway}

The outcomes of HCV antibody positive inmates seen in the HWC are shown in figure 2. Of the 41 inmates who had evidence of ongoing HCV infection, only six (15\%) were deemed eligible for treatment, the main reasons for ineligibility being psychiatric illness, persisting high risk behaviours, and failure to biopsy during their prison term. Only three patients have undergone treatment over the two years since the start of the study due to failure of the funding body to support this NICE approved therapy.

Eligibility for liver biopsy and treatment rates of HCV RNA positive patients of $41 \%$ and $15 \%$, respectively, compare with values of $80 \%$ and $50 \%$ for the local adult hepatology outpatient clinic.

\section{Impact of healthwatch and prison outreach clinics}

Prior to instigation of the HWC, approximately five patients each year were referred to Southampton University Hospitals Trust for investigation of hepatitis C. During the 16 months following the establishment of the HWC prior to instigation of the POC, 78 prisoners were referred as outpatients to Southampton University Hospitals Trust. During the study period, 17 prisoners had a consultant appointment in the POC and seven biopsies were performed as inpatients at Southampton General Hospital.

\section{DISCUSSION}

The National Strategy for Hepatitis C recognises that prisons represent a controlled environment within which treatment for HCV can be offered to a population with a high prevalence of infection and in which risk factors for disease progression, such as heavy alcohol consumption and smoking, are all prominent. It is difficult to determine with accuracy the national prevalence of IDU but it is estimated that there are 100-200 000 IDU with serious dependency in the UK. ${ }^{10}$ Due to the criminalisation of drug use and the relationship between drug dependency and crime, many IDU will serve a prison sentence. Although prisoners are frequently moved between prisons and many serve short sentences, 58\% of prisoners in England and Wales discharged in 1997 were convicted of a standard list offence within the next two years, providing the opportunity for healthcare interventions. Despite a high prevalence of HCV infection, this study found that the rate of uptake of voluntary testing among new inmates was low and only a minority proceeded to treatment. 
Table 2 Uptake of hepatitis $\mathrm{C}$ virus (HCV) testing in the healthwatch clinic and prevalence of positive HCV tests

\begin{tabular}{llllll}
\hline Prison & $\begin{array}{l}\text { No of } \\
\text { receptions }\end{array}$ & $\begin{array}{l}\text { Tests } \\
\text { requested }\end{array}$ & $\begin{array}{l}\text { HCV antibody } \\
\text { positive (\%) }\end{array}$ & $\begin{array}{l}\text { HCV PCR positive } \\
(\%)\end{array}$ & \% HCV PCR/Ab positive \\
\hline Parkhurst & 610 & 40 & $19(47.5)$ & $15(37.5)$ & 78.9 \\
Camp Hill & 800 & 93 & $35(37.6)$ & $23(24.7)$ & 65.7 \\
Albany & 208 & 4 & $4(100)$ & $3(75)$ & 75 \\
Total & 1618 & $137(8.5)$ & $58(42)$ & $41(29.9)$ & 70.7 \\
\hline
\end{tabular}

The number and percentage of prisoners accepting testing for $\mathrm{HCV}$, testing positive for $\mathrm{HCV}$ antibodies in serum, and testing positive for HCV nucleic acid by polymerase chain amplification (PCR) are presented.

$\mathrm{Ab}$, antibody.

Despite having an outreach service that provided counselling on hepatitis C from experienced health professionals, uptake was less than $10 \%$. Clearly, this limits the effectiveness of any strategy to reduce the burden of hepatitis C. Increasing awareness of the condition and the effectiveness of therapy may encourage more inmates to accept testing. Uptake of testing was highest in prisons that received the greatest number of prisoners sentenced for drug offences, suggesting that inmates appropriately identified their suitability for testing following the initial general health lecture at the time of reception. It is likely that the prevalence of hepatitis $\mathrm{C}$ was high among the $90 \%$ of new inmates who did not attend the HWC. The National Strategy stresses the need to increase awareness of HCV and the HWC can be assumed to have achieved this, although we have no confirmatory data and cannot determine its effect.

Hepatitis C seropositivity at $30 \%$ was considerably higher than the $9 \%{ }^{11}$ or $7 \%{ }^{7}$ reported in earlier studies in the UK but comparable with the $37 \%$ found by anonymous testing in Irish prisons. ${ }^{8}$ IDU was the predominant risk factor. The prevalence of HCV among IDU in the Irish study was $81 \%$ while that in the UK study of Weild and colleagues ${ }^{7}$ was $30 \%$. We found that $96 \%$ of HCV positive inmates had a history of IDU but we do not know the prevalence of IDU among inmates who did not attend the HWC. Of concern was the high percentage of prisoners in our study $(33 \%)$ who admitted to having injected while in prisons, suggesting that measures to contain injecting drug use in prisons are failing. Given the high prevalence of HCV in the prison population, prisoners and exprisoners will continue to form a major reservoir for transmission of infection. Other risk factors reported included sharing toothbrushes and razors.

A striking finding was the high frequency with which prisoners had been recipients of blood transfusion, which was 80 times the rate of blood transfusion in the general population $(0.1 \%) .{ }^{12}$ This may reflect a high rate of violence and trauma. Specific measures directed at restricting the transmission of infection in prisons are therefore required. Prisons provide an ideal opportunity for disseminating accurate healthcare information concerning risk factors,

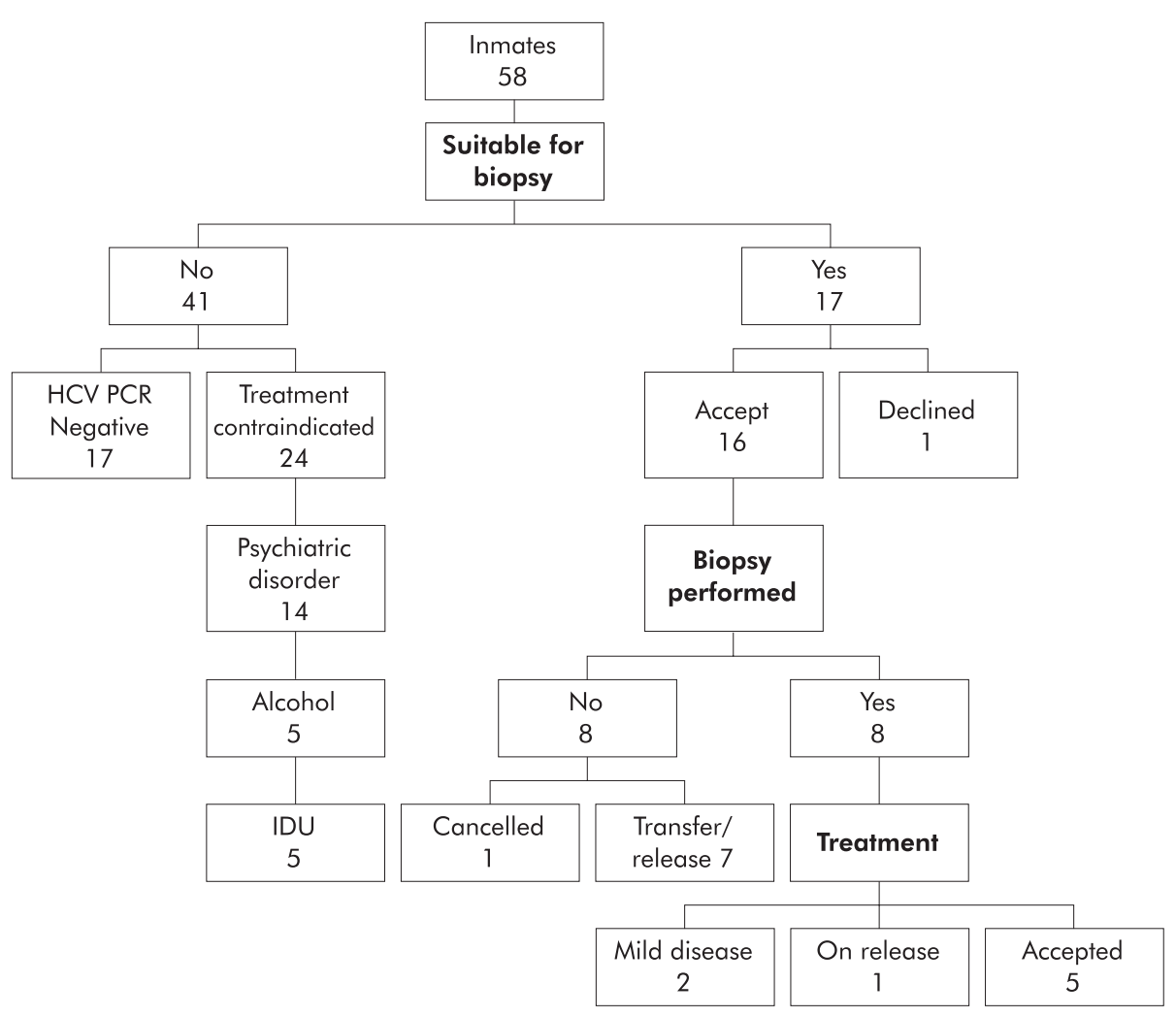

Figure 2 Outcome of prisoners accepting testing. The outcomes for the 58 prisoners who tested positive for hepatitis $\mathrm{C}$ virus (HCV) antibodies in the prison outreach clinic. Prisoners were managed according to the algorithm shown in figure 1. PCR, polymerase chain amplification; IDU, injecting drug users. 
natural history, and harm minimisation among both those already infected with HCV and those not infected but at very high risk of exposure.

This study found a very high level of ineligibility for treatment in those who had signs of active infection, with 25 of $41(61 \%)$ being ineligible due to a contraindication. In the majority of cases, this was because of a past or current medical history of serious psychiatric disorder. In the remainder, explicit choices to continue drug or alcohol misuse on release rendered them ineligible. This accords with current guidance. The overwhelming majority of experience of the treatment of hepatitis $\mathrm{C}$ has been in the context of clinical trials in which patients with psychiatric illness have largely been excluded due to the propensity for interferon alpha to aggravate psychiatric illness, particularly depression. Ongoing psychiatric illnesses are common, with some reports as high as $75 \%$. Outside prison those with varying degrees of minor psychiatric illness are being offered treatment, although with additional support from psychiatrists and psychiatric nurses at some cost. Development of such strategies may well permit more widespread treatment of patients currently considered to be ineligible. The feasibility, effectiveness, and costs of improved psychiatric care in the prison setting have not been studied.

During their sentence, 9/17 patients deemed eligible for treatment did not undergo liver biopsy while in prison, which is currently required for histological proof of suitability for antiviral therapy..$^{13}$ Nine inmates refused a biopsy despite knowing that this would exclude them from therapy; this is an uncommon choice in the general outpatient setting. Eight opted to defer further management until after they had been released, two of whom were referred directly to local hepatologists and have begun treatment in their home towns. No formal process exists for referral from the prison health services to local NHS services and such a process should be accommodated in the managed clinical networks advocated by the National Strategy.

The relatively lower rates of liver biopsy in the prison population compared with the general clinic setting reflect both logistic difficulties in arranging for prisoners to have biopsies and the choices of prisoners. Maintaining the secure containment of prisoners during biopsy necessitates prison officers accompanying the inmates on visits to the hospital and during inpatient stays. This is costly and could be circumvented by performing biopsies in the prison but this would require a significant increase in the provision of diagnostic, nursing, and medical care. More prisoners deferred or declined biopsy than is usual in the outpatient clinic but these choices may be appropriate to the inmates' understanding of their current circumstances.

The HWC is an innovative service that has allowed us to determine the prevalence of HCV infection and to undertake in the prisons a major part of the secondary care of patients referred with hepatitis C. During the study period, over 70 new patients that would have been referred to the general hepatology outpatient clinic were managed in the prisons. This relieved pressure on the hospital as well as dramatically reducing the demands on the time of prison officers accompanying inmates to clinic appointments and hospital admissions. The POC has resulted in considerable financial savings for both prisons and the hospital trust. Both the healthwatch and outreach clinics have increased the level of confidentiality for prisoners with blood borne virus infection. They have also improved the quality of information disseminated among prisoners regarding blood borne viruses and particularly hepatitis $\mathrm{C}$ that was frequently confused with HIV within the prison cluster prior to establishment of the clinics. However, despite the provision of confidential testing and a care pathway, there was little impact on the burden of infection in this high risk group. They are at high risk of transmitting the infection both in prison and after discharge, and of complications. New strategies directed at reducing transmission and harm are needed to deal with this, along with the development of therapies that incur fewer of the adverse effects that limit the use of antiviral drugs in this population.

\section{ACKNOWLEDGEMENTS}

The authors wish to thank Mr Karl Chads at the Home Office for providing additional data on the ethnicity of prisoners in the Isle of Wight prisons.

\section{Authors' affiliations}

C Skipper, HMP Parkhurst, Isle of Wight, UK

J M Guy, Liver Group, Division of Infection, Inflammation, and Repair, University of Southampton, Southampton, UK

J Parkes, P Roderick, Health Services Research Unit, Liver Epidemiology Group, Division of Community Clinical Sciences, University of

Southampton, Southampton, UK

W M Rosenberg, Liver Group, Division of Infection, Inflammation, and Repair, University of Southampton, and Health Services Research Unit, Liver Epidemiology Group, Division of Community Clinical Sciences, University of Southampton, Southampton, UK

\section{REFERENCES}

1 Department of Health. Hepatitis C Strategy for England, 2002. http:// www.doh.gov.uk/cmo/hcvstrategy/77097dhhepcstrat.pdf (accessed 14 July 2003).

2 Atrah HI, Ala FA, Ahmed MM, et al. Unexplained hepatitis C virus antibody seroconversion in established blood donors. Transfusion 1996;36:339-43.

3 Ward C, Tudor-Williams G, Cotzias T, et al. Prevalence of hepatitis C among pregnant women attending an inner London obstetric department: uptake and acceptability of named antenatal testing. Gut 2000;47:277-80.

4 Crofts N, Hopper JL, Milner R, et al. Blood-borne virus infections among Australian injecting drug users: implications for spread of HIV. Eur J Epidemiol 1994; 10:687-94

5 Goldberg D, Cameron S, McMenamin J. Hepatitis C virus antibody prevalence among injecting drug users in Glasgow has fallen but remains high. Commun Dis Public Health 1998;1:95-7.

6 http://www.hmprisonservice.gov.uk/ (accessed 14 July 2003).

7 Weild AR, Gill ON, Bennett D, et al. Prevalence of HIV, hepatitis B, and hepatitis $C$ antibodies in prisoners in England and Wales: a national survey. Commun Dis Public Health 2000;3:121-6.

8 Allwright S, Bradley F, Long J, et al. Prevalence of antibodies to hepatitis B, hepatitis $C$, and HIV and risk factors in Irish prisoners: results of a national cross sectional survey. BMJ 2000;321:78-82.

9 Thornton L, Barry J, Long J, et al. Comparison between self-reported hepatitis B, hepatitis C, and HIV antibody status and oral fluid assay results in Irish prisoners. Commun Dis Public Health 2000;3:253-5.

10 Wiessing L, Hartnoll R. European Monitoring Centre for Drugs and Drug Addiction (EMCDDA). Study to obtain comparable national estimates for problem drug use prevalence for all EU member states. Lisbon: EMCDDA, 1999.

11 Marshall T, Simpson S, Stevens A. Health care needs assessment in prisons: a toolkit. J Public Health Med 2001 ;23:198-204.

12 Mutimer DJ, Harrison RF, O'Donnell KB, et al. Hepatitis C virus infection in the asymptomatic British blood donor. J Viral Hepat 1995;2:47-53.

13 Booth JC, O'Grady J, Neuberger J. Clinical guidelines on the management of hepatitis C. Gut 2001;49(suppl 1):11-21.

14 National Institute of Clinical Excellence. Guidance on the use of ribavirin and interferon alpha for the treatment of hepatitis C. Guidance No. 14. London: National Institute of Clinical Excellence, 2000. 